\title{
Seroprevalence of Dengue by Detection of NS1, IgM in Tertiary Care Hospital, RIMS, Ranchi
}

\author{
Vidushi Topno', Manoj Kumar ${ }^{2}$, Ashok Kumar Sharma ${ }^{3}$, Kumari Seema ${ }^{4}$ \\ ${ }^{1}$ Post Graduate, ${ }^{2}$ Professor, ${ }^{3}$ Associate Professor, ${ }^{4}$ Assistant Professor, \\ Department of Microbiology, RIMS, Ranchi \\ Corresponding Author: Manoj Kumar
}

DOI: https://doi.org/10.52403/ijrr.20220220

\begin{abstract}
Introduction: Dengue virus being most significant among vector borne diseases is leading to increased morbidity and mortality in India. Global prevalence of dengue has been increasing over the last decade. So, it is important to screen all dengue fever like cases.

Objective: The study was conducted to know seroprevalence of Dengue virus by detecting NS1, IgM in clinically suspected dengue fever cases.

Material and Method: Test was done in 315 blood samples of clinically suspected dengue fever cases, in various departments of RIMS, Ranchi. The study was done from November 2016 to August 2017. NS1 antigen and IgM antibody detection both were done by ELISA (NS1 by Qualisa Microwell Enzyme Immunoassay and IgM by NIV Dengue IgM Captured ELISA KIT).

Result and Discussion: Out of 315 blood samples, 84 samples $(26.7 \%)$ were serologically positive for dengue infection. Among the positive cases $72.6 \%$ were males and $27.4 \%$ were females. Males are more affected than females, because males are more exposed during their outdoor visit for livelihood. Age group between 21-30 years were most affected. Most of the cases were reported in monsoon and postmonsoon season.

Conclusion: This study highlights the need of continuous surveillance by both NS1 antigen and IgM antibody by ELISA method for early detection of impending outbreak to initiate timely prevention and control measures.
\end{abstract}

Keywords: Dengue, NS1, IgM

\section{INTRODUCTION}

Dengue is a mosquito borne disease and transmitted mainly by Aedes aegypti and Aedes albopictus mosquito. ${ }^{1}$ Wide range of symptoms are seen in infection ranging from mild non-specific fever to more severe and lethal dengue hemorrhagic fever (DHF) and dengue shock syndrome (DSS)2. It is caused by virus Flaviviridae. There are four serotypes of the virus referred to as DV-1, DV-2, DV-3 and DV-4. It is a positivestranded encapsulated RNA virus with three structural protein genes. Genes encode the nucleocapsid or core (C) protein, a membrane-associated (M) protein, an enveloped (E) glycoprotein and seven nonstructural (NS) proteins.1

According to classification of WHO 2009 dengue fever is classified into two groups: uncomplicated and severe ${ }^{3}$, but still the 1997 WHO classification is widely used $^{4}$. Dengue is classified into undifferentiated fever, dengue fever (DF), and dengue haemorrhagic fever (DHF) according to the 1997 classification. ${ }^{3}$ There are four main characteristic manifestations of dengue illness, $(i)$ continuous high fever lasting 2-7 days; (ii) thrombocytopaenia (platelet count $<100 \times 10^{9} / 1$ ); (iii) haemorrhagic tendency as shown by a positive tourniquet test, petechiae or epistaxis; and (iv) evidence of plasma leakage manifested by haemoconcentration (an increase in haematocrit $20 \%$ above average for age, sex and population), ascites and pleural effusion and, etc. ${ }^{1}$ 
In routine laboratories Dengue IgM and IgG ELISA kits are widely used for diagnosis of dengue infection. Variations are there in detection limit during acute phase of the disease. Usually it takes 4-5 and 1-14 days respectively after the onset of symptoms for anti-DENV $\operatorname{IgM}$ and $\operatorname{IgG}$ antibodies to become detectable. But, it depends whether the patient has primary or secondary infection. ${ }^{5}$ The gold standard for diagnosis of acute cases is isolation of virus in cell culture or in infant mouse. This requires specialized laboratories which takes more than a week for the test to be completed. In most of the situation it becomes impractical. Early diagnosis during febrile phase can be detection of viral RNA by RT-PCR. The procedure is cumbersome, in case of RT-PCR with difficult interpretation lack of immediate result thus the procedure becomes difficult. ${ }^{6}$ In laboratory, during the late incubation period, or initial phase of dengue virus infection, for disease confirmation detection is done by viral isolation in cell culture and/or molecular investigations, or immunohistochemistry immunofluorescence. ${ }^{7}$

For detection of dengue virus in the early stages of the disease there is need for rapid, sensitive methods.6 Right at the beginning of the feverish period and before the appearance of dengue IgM and/or IgG, non-structural antigen non-structural protein 1 (NS1) develops. ${ }^{8}$

During the first few days of illness, ELISA assays is used in combination of NS1 and IgM detection in samples. It was recommended to increase overall dengue diagnostic sensitivity. ${ }^{9}$

Platelet therapy is used in standard clinical practice for dengue patients with severe thrombocytopenia. ${ }^{10}$ Platelet count is not done as introductory screening, in many cases. This results in delays in starting platelet therapy. In New Delhi, during the 2010 spurt in the incidence of dengue, a simultaneous screening for NS1, IgM and IgG and platelet enumeration was launched at the Sant Parmanand Hospital. ${ }^{11}$
In state of Jharkhand, RIMS is one of the best tertiary care institutes. Department of Microbiology, in RIMS, Ranchi caters in diagnosis wide range of vector-borne diseases and other infectious diseases. The study aims to know seroprevalence of Dengue virus by detecting NS1, IgM in clinically suspected dengue fever cases.

\section{MATERIAL AND METHODS}

This retrospective study was conducted in the department of Microbiology (Virology) RIMS, Ranchi. Study period was from November 2016 to August 2017. Total 315 blood samples of clinically suspected cases of dengue fever were collected from both outdoor and hospitalised patient from RIMS, Ranchi. Patient's details were taken from data recorded in Department of Microbiology, RIMS, Ranchi. After standard separation of serum, samples were analysed for the detection of NS1 antigen and IgM antibodies. Both the tests were done by ELISA method (NS1 by Qualisa Microwell Enzyme Immunoassay) and (IgM by NIV Dengue IgM captured ELISA Kit. Manufacturer's instructions were strictly followed for performing the test and interpreting the results.

\section{RESULT}

A total of 315 blood samples were tested from suspected dengue fever cases. Of these 84 samples $(26.7 \%)$ were serologically positive to have dengue infection. Out of 84 samples 14 (16.70\%) were NS1 positive and $59(70.20 \%)$ were positive for IgM. Among them 11(13.10\%) were positive for both NS1 and IgM. Among the positive dengue infection patients, male patients were $61(72.6 \%)$ and female patients were $23(27.4 \%)$. Male dominance was seen among positive patients. 


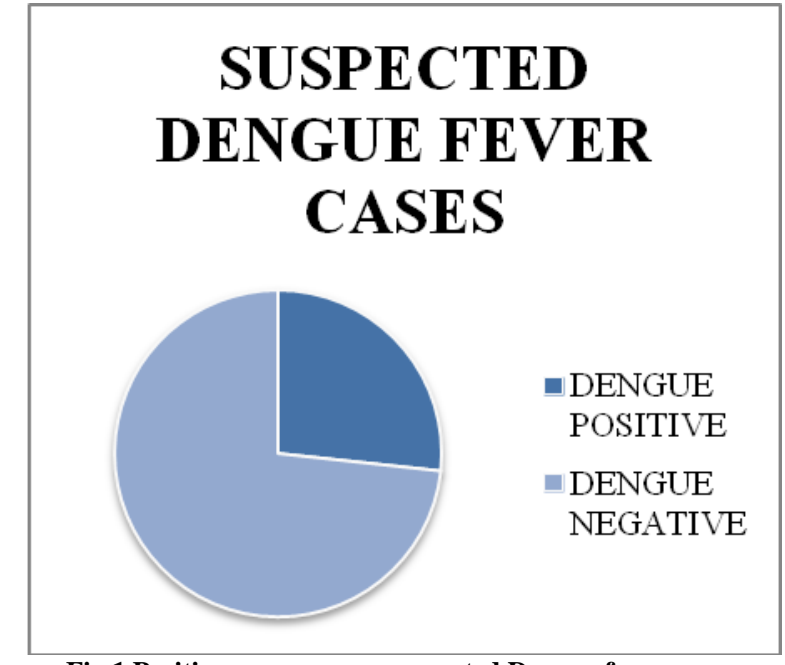

Fig.1 Positive cases among suspected Dengue fever cases

Table 1: NS1 antigen in Dengue samples

\begin{tabular}{|l|l|l|}
\hline NS1 Antigen & No. of patients & Percentage \% \\
\hline Only NS1 & 14 & $16.70 \%$ \\
\hline NS1 + IgM & 11 & $13.10 \%$ \\
\hline NS1 negative & 59 & $70.20 \%$ \\
\hline
\end{tabular}

\section{DENGUE POSITIVE CASES}

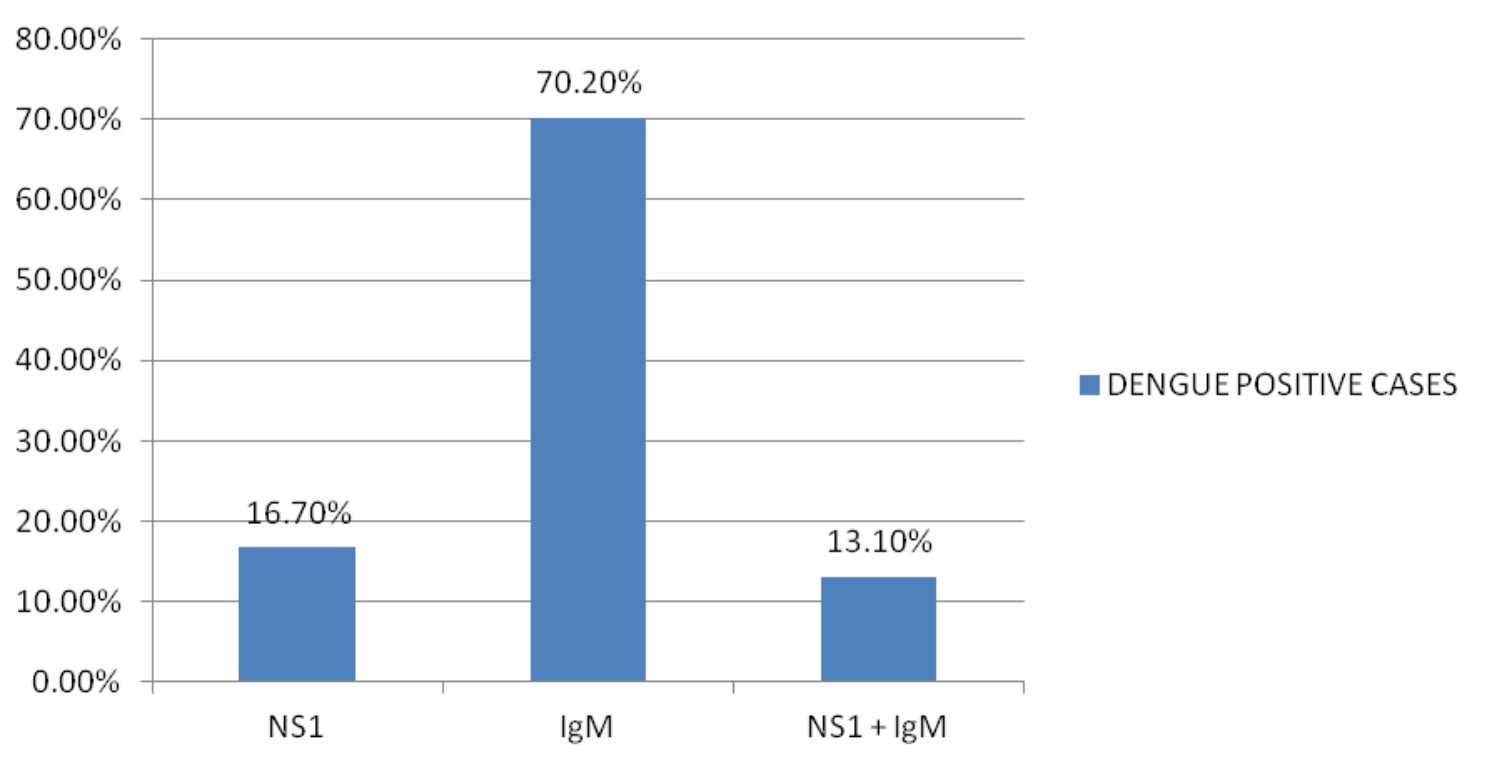

Fig.3 Presence of NS1 positive and IgM positive cases among Dengue positive cases

Age group between $10-65$ years were affected. The age group affected the most was 21-30 years. Most of the cases were reported in monsoon and postmonsoon season.

\section{DISCUSSION}

Patients with NS1 positive were $14.73 \%$. There were $62.10 \%$ of positive cases which showed only $\operatorname{IgM}$ antibodies
Table 2 : IgM antibodies in Dengue samples

\begin{tabular}{|l|l|l|}
\hline IgM antibody & No. of patients & Percentage\% \\
\hline Only IgM & 59 & $70.20 \%$ \\
\hline IgM with NS1 & 11 & $13.10 \%$ \\
\hline IgM negative & 14 & $16.70 \%$ \\
\hline
\end{tabular}

\section{Male Female Ratio}

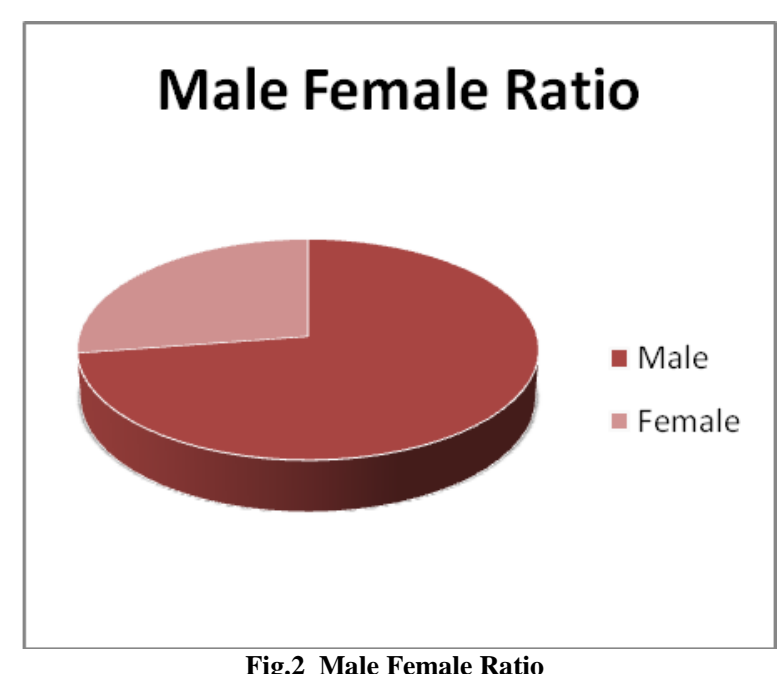

Fig.2 Male Female Ratio 
followed by appearance of only $\operatorname{IgM}$ antibodies. Present study reveals significant role of dengue serology in early diagnosis of dengue. Implying that the combination of these serological tests would increase the rate of detection of dengue fever. We found that the age group affected the most was 2130 years. Study showed male predominance in Dengue infection cases (72.6\%).

Study highlights continuous surveillance by dengue serology for early detection of impeding outbreak, to initiate timely prevention and control method. Due to cross reactivity with other flaviviruses IgM detection is not always conclusive ${ }^{12}$. Dengue RT-PCR use in most laboratories is currently difficult, because of the cumbersome procedure, prolonged time and difficult interpretation. ${ }^{13-15}$

For all dengue serotypes NS1 protein is highly conserved. It is circulating in high levels during the first few days of illness. No cross-reaction of dengue NS1 protein with those of other related flaviviruses is seen. ${ }^{16}$ The detection of plasma NS1 would be associated with plasma viremia levels. NS1 is the product of infected cells. It is reported in Srivastava et al that in patients who were NS1-positive, viremia levels were significantly higher when compared to those who were NS1negative. ${ }^{17}$ In another study in dengue-2 virus the level of NS1 in plasma was correlated with viremia levels. Within $72 \mathrm{~h}$ of onset of illness, the level was significantly higher in patients with dengue hemorrhagic fever when compared with patients of dengue fever ${ }^{18}$. The nested RTPCR developed by Lanciotti et al ${ }^{19}$, and it's modification by Harris et al is the most widely used PCR based method for detecting DENV nucleic acid in the serum. ${ }^{20}$ This test is having high sensitivity and specificity, and that reaction set-up to result time that is much shorter as in recent literature ${ }^{21-23}$. Due to formation of immune complexes there can be decreased levels of NS1 after 4 days of illness thus monoclonal antibodies does not assess the target epitopes. ${ }^{24,25}$

\section{CONCLUSION}

Controlling dengue infection is becoming challenging because it requires effective vector control. Dengue NS1 has gained considerable interest as biomarker for early diagnosis of dengue illness. ELISA based NS1 and IgM antibody detection is more efficient and popular due to its simplicity, high specificity and great sensitivity.

Acknowledgement: None

Conflict of Interest: None

Source of Funding: None

Ethical Approval: Approved

\section{REFERENCES}

1. Gupta N, Srivastava S, Jain A, Chaturvedi UC. Dengue in India. Indian J Med Res. 2012 Sep;136(3):373-90. PMID: 23041731; PMCID: PMC3510884.

2. Whitehead SS, Blaney JE, Durbin AP, Murphy BR. Prospect for a dengue virus vaccine. Nat Rev Microbiol 2007; 5: 518-28.

3. Whitehorn J, Farrar J. Dengue. Br Med Bull. 2010;95:161-

73. [PubMed] [Google Scholar]

4. Dengue: Guidelines for diagnosis, treatment, prevention, and control in sub-Saharan Africa and 13 countries in South America. Geneva: World Health Organization; 2009. WHO. [Google Scholar]

5. Schilling S, Ludolfs D, Van An L, Schmitz H. Laboratory diagnosis of primary and secondary dengue infection. J Clin Virol 2004; 31(3): 179-84.

6. Ahmed and Broor: Rapid diagnosis of dengue infection in acute phase $\mathrm{J}$ Vector Borne Dis 51, September 2014

7. Centers for Disease Prevention and Control. Dengue: Laboratory criteria for diagnosis for case definitions. From http://www.cdc.gov/dengue/ 
clinicalLab/caseDef.html Accessed Sep 10

8. Thomas L, Najioullah F, Verlaeten O, Martial J, Brichler S, Kaidomar S, et al. Relationship between nonstructural protein 1 detection and plasma virus load in Dengue patients. Am J Trop Med Hyg 2010; 83:696-9.

9. Guzman MG, Jaenisch T, Gaczkowski R. Multi-country evaluation of the sensitivity and specificity of two commercially-available NS1 ELISA assays for dengue diagnosis. PLoS Negl Trop Dis 2010; 4:e811.

10. Thomas L, Kaidomar S, Kerob-Bauchet B, Moravie V, Brouste Y, King JP, et al. Prospective observational study of low thresholds for platelet transfusion in adult dengue patients. Transfusion 2009; 49:1400-11. Epub 20 Mar 2009.

11. BBC News South Asia. Disease threat at Delhi Commonwealth Games site. From: http://www. bb c .co.uk/ne ws/world- south-a sia-11241218. Accessed: Sep 2010.

12. Dengue haemorrhagic fever: Diagnosis, treatment and control.Geneva: World Health Organization 1997. Available from:http://www.who.int/csr/resources/p ublications/dengue/Denguepublication/e n/inbex.html

13. Lanciotti RS, Calisher CH, Gubler DJ, Chang GJ, Vorndam AV. Rapid detection and typing of dengue viruses from clinical samples by using reverse transcriptase-polymerase chain reaction.J Clin Microbiol 1992; 30: 54551.

14. Harris E, Roberts TG, Smith L, Selle J, Kramer LD, Valle S, etal. Typing of dengue viruses in clinical specimens and mosquitoes by single-tube multiplex reverse transcriptase PCR. J Clin Microbiol 1998; 36: 2634-9.

15. Shu PY, Huang JH. Current advances in dengue diagnosis. Clin Diagn Lab Immunol 2004; 11: 642-50.

16. Lapphra K, Sangcharaswichai A, Chokephaibulkit K, TiengrimS, Piriyakarnsakul W, Chakorn T, et al,
Evaluation of an NS1antigen detection for diagnosis of acute dengue infection in patients with acute febrile illness. Diagn Microbiol Infect Dis 2008;60: 387-91.

17. Srivastava A, Dash PK, Tripathi NK, Sahni AK, Gopalan N,Lakshmana Rao $\mathrm{PV}$. Evaluation of a commercial dengue Correspondence to: Dr Shobha Broor, Professor, Department of Microbiology, SGT Medical College-Hospital and Research Institute, Gurgaon-122 505 (Haryana), India.E-mail: shobha.broor@gmail.com

18. Xu H, Di B, Pan YX, Qiu LW, Wang YD, Hao W, et al. Serotype1-specific monoclonal antibody-based antigen capture immunoassay for detection of circulating nonstructural protein NS1: implications for early diagnosis and serotyping of denguevirus infections. J Clin Microbiol 2006; 44: 2872-8.

19. Lanciotti RS, Calisher CH, Gubler DJ, Chang GJ, Vorndam AV. Rapid detection and typing of dengue viruses from clinical samples by using reverse transcriptase-polymerase chain reaction.J Clin Microbiol 1992; 30: 54551.

20. Harris E, Roberts TG, Smith L, Selle J, Kramer LD, Valle S, et al. Typing of dengue viruses in clinical specimens and mosquitoes by single-tube multiplex reverse transcriptase PCR. J Clin Microbiol 1998; 36: 2634-9.

21. Saxena P, Dash PB, Santhosh SR, Srivastava A, Parida M, Rao PVL. Development and evaluation of one step single tube multiplex RT-PCR for rapid detection and typing of dengue viruses.Virol J 2008, 5: 20 .

22. Waggoner JJ, Abeynayake J, Sahoo MK, Gresh L, Tellez Y, Gonzalez K, et al. Single-reaction, multiplex, real-time RT-PCR for the detection, quantitation, and serotyping of dengue viruses. PLoS Negl Trop Dis 2013; 7(4): e2116.

23. Mishra B, Sharma M, Pujhari SK, Appannanavar SB, Ratho RK. Clinical 
applicability of single-tube multiplex reverse-transcriptase PCR in dengue virus diagnosis and serotyping. J Clin Lab Anal 2011; 25(2): 76-8.

24. Alcon S, Talarmin A, Debruyne M, Falconar A, Deubel V, Flamand M. Enzyme-linked immunosorbent assay specific to dengue virus type 1 nonstructural protein NS1 reveals circulation of the antigen in the blood during the acute phase of disease in patients experiencing primary or secondary infections. J Clin Microbiol 2002; 40: 376-81.

25. Shu PY, Chen LK, Chang SF, Yueh YY, Chow L, Chien LJ,et al. Potential application of nonstructural protein NS1 serotype- specific immunoglobulin G enzyme-linked immunosorbent assay in the seroepidemiologic study of dengue virus infection: correlation of results with those of the plague reduction neutralization test. J Clin Microbiol 2002; 40: 1840-4.

How to cite this article: Topno V, Kumar M, Sharma AK et.al. Seroprevalence of dengue by detection of NS1, IgM in Tertiary Care Hospital, RIMS, Ranchi. International Journal of Research and Review. 2022; 9(2): 137-142. DOI: https://doi.org/10. 52403/ijrr.20220220 